Published in final edited form as:

Curr Opin Urol. 2012 July ; 22(4): 320-327. doi:10.1097/MOU.0b013e32835483d5.

\title{
Molecular Imaging of Prostate Cancer
}

\author{
Josef J. Fox ${ }^{1}$, Heiko Schöder ${ }^{1}$, and Steven M. Larson ${ }^{1}$ \\ ${ }^{1}$ Molecular Imaging and Therapy Service, Department of Radiology, Memorial Sloan-Kettering \\ Cancer Center, New York, New York
}

\begin{abstract}
Purpose of review-Prostate cancer is a complex and biologically heterogeneous disease that is not adequately assessed with conventional imaging alone. Molecular imaging with positron emission tomography (PET) is poised to fill this unmet need through noninvasive probing of the multiple molecular and cellular processes that are active in prostate cancer patients.
\end{abstract}

Recent findings-Several PET tracers are active in early and late stage prostate cancer in humans. F18-FDG, C11/F18-choline and F18-sodium fluoride (NaF) have been studied most extensively. There is a growing body of literature supporting to the utility of choline in early stage prostate cancer. FDG and NaF are more valuable in advanced disease, especially for assessing bone metastases, the prevalent form of metastases in this patient population. F18-Fluorodihydrotestosterone is active in castrate disease and is emerging as a valuable pharmacodynamic marker in the development of novel AR-targeted therapies. Anti-PSMA PET tracers are in the early stages of clinical development.

Summary-Multiple PET tracers are currently available to aid in the detection and management of prostate cancer across the clinical spectrum of the disease. Prospective, rigorously controlled, clinical imaging trials are needed to establish the optimal role of PET in prostate cancer.

\section{Keywords}

Prostate cancer; PET; FDG; Choline; FDHT

\section{Introduction}

Prostate cancer is a biologically heterogeneous disease across the spectrum of its clinical states $^{1}$, and is thus challenging to treat. Successful management is further confounded by the disease-specific limitations of conventional imaging ${ }^{2}$. Positron emission tomography (PET) carries a distinct advantage over conventional imaging in its unique ability to non-invasively interrogate metabolic and molecular processes in-vivo. These processes include alterations in glucose, amino acid and fatty acid metabolism, receptor status, cellular proliferation, tumor hypoxia and blood flow. Several PET tracers are active in early and late stage prostate cancer (see Table 1). Among these, F18-FDG, Choline (C11 and F18 labeled) and sodium

Corresponding Author: Josef J. Fox, M.D., Memorial Sloan-Kettering Cancer Center, 1275 York Ave., New York, NY 10065, Tel: 212-629-7371 Fax: 212-717-3263 foxj@mskcc.org. 
F18-fluoride ( $\mathrm{NaF}$ ) have been studied most extensively. Choline is emerging from the vast cloud of data (quite heterogeneous, akin to the disease itself) as a valuable tool for assessment of early prostate cancer, while FDG and $\mathrm{NaF}$ appear to have greater utility in advanced disease.

\section{C11 and F18 Choline}

The two radiolabeled forms of choline are generally considered to provide similar information, with the advantage of $\mathrm{C} 11$ labeled choline being minimal urinary excretion, while the F18 compound is better fit for commercial use, owing to its longer half life (110 minutes vs 20 minutes for C11). Choline PET is increasingly utilized in the Eastern Hemisphere for evaluation of prostate cancer; however, in many studies the patient populations and states of disease are quite heterogenous. A recent systematic review ${ }^{3}$ of 37 studies noted several sources of variation affecting the reported performance of F18-choline PET, underscoring the need for rigorously standardized prospective imaging trials. Nonetheless, it is apparent that choline PET is a rational and viable modality for evaluating prostate cancer, particularly in its early stages.

A major challenge for PET imaging of localized prostate cancer is the difficulty in detecting small lesions and in discriminating between benign and malignant processes. This is a wellknown challenge for FDG, but also exists for other tracers including choline-PET. In an elegant study, Souvatzoglou and colleagues recently evaluated 43 patients who underwent radical prostatectomy within 31 days after $\mathrm{C} 11$-choline PET/CT ${ }^{4}$. Transaxial images and histologic specimens were analyzed by comparing the respective slices. Not surprisingly, the authors found that small focal tumors $(<5 \mathrm{~mm})$ and rind-like tumors were poorly detected on PET, while larger well-defined tumors were evident on PET (figure 1). In addition, SUVmax failed to distinguish between cancer (median SUVmax 4.9), benign prostatic hypertrophy (median SUVmax 4.5) and prostatitis (median SUVmax 3.9), $\mathrm{P}=0.102$ and $\mathrm{P}=$ 0.054 , respectively.

The greatest value of the choline agents seems to be in the setting of rising PSA following definitive local therapy. By definition, patients with "biochemical relapse" have a rising PSA level as the only manifestation of their disease ${ }^{5}$. The goal of molecular imaging with PET in this setting is to localize foci of recurrent disease that are unrecognized clinically or on conventional imaging (e.g., small lymph nodes that do not meet size criteria on standard CT or MRI, or disease in bone marrow that is not recognizable on CT). The main purpose here is to distinguish between local, regional or distant disease recurrence, because the treatment will vary (e.g., irradiation to prostate bed versus hormonal or chemotherapy). Choline uptake in prostate cancer is presumed to correlate with tumor volume as reflected by PSA levels. In a report on 63 patients categorized in biochemical recurrence, 35 (56\%) patients had abnormal scans ${ }^{6}$. Recurrent disease could be localized in $36 \%$ of patients with PSA-value $<1 \mathrm{ng} / \mathrm{ml}, 43 \%$ with PSA-value 1 to $<2 \mathrm{ng} / \mathrm{ml}, 62 \%$ with PSA-value 2 to $<3$ $\mathrm{ng} / \mathrm{ml}$ and $73 \%$ with PSA-value $\geq 3 \mathrm{ng} / \mathrm{ml}$. The detection rate was not influenced by concurrent anti-androgen therapy. Other investigators have demonstrated a strong link between PSA kinetics - including PSA doubling time (PSADT) and velocity (PSAV) - and abnormal choline PET/CT findings. Shorter doubling time and higher PSA velocity reflect 
the rate of tumor cell proliferation. Giovacchini and colleagues ${ }^{7}$ studied 170 patients with biochemical relapse following radical prostatectomy, C11-choline PET/CT was positive in 75 of 170 patients (44\%). The percentage of patients with positive C11-choline PET/CT was $27 \%$ for PSADT $>6$ months, $61 \%$ for PSADT between 3 and 6 months and $81 \%$ for PSADT $<3$ months. Pathologic uptake in the skeleton was seen in $52 \%$ of patients with PSADT $<3$ months and only in $3 \%$ of patients with PSADT $>6$ months. More recently, the same group showed a positive correlation with PSAV in the same patient population ${ }^{8}$. Patients with positive C11-choline PET/CT $(\mathrm{n}=75)$ had significantly $(\mathrm{p}<0.05)$ higher PSAV than patients with negative scans $(\mathrm{n}=95)(6.93 \pm 13.08 \mathrm{vs} .1 .23 \pm 2.03 \mathrm{ng} / \mathrm{mL} / \mathrm{y})$. The percent of patients with positive C11-choline PET/CT was $21 \%$ for PSAV $<1 \mathrm{ng} / \mathrm{mL} / \mathrm{y}, 56 \%$ for PSAV between 1 and $2 \mathrm{ng} / \mathrm{mL} / \mathrm{y}$, and $76 \%$ for PSAV $>2 \mathrm{ng} / \mathrm{mL} / \mathrm{y}$. These and other studies ${ }^{9,10}$ clearly demonstrate a relationship between choline uptake and PSA kinetics; however, it is worth noting that the patient populations studied do not strictly qualify for "biochemical relapse", as many of the PET-positive lesions were also present on conventional imaging.

\section{F18-Sodium Flouride (NaF)}

Bone metastases are the primary cause of morbidity and mortality in progressive prostate cancer, eventually developing in $80-90 \%$ of patients with metastatic disease, either alone or in conjunction with less frequent visceral metastases ${ }^{11}$. Bone disease is notoriously difficult to assess by conventional radiography and computed tomography. In fact, the osteoblastic variety, most commonly found in prostate cancer patients, is considered non-measurable disease according to RECIST ${ }^{12}$. Scintigraphic techniques have long been the mainstay for assessing bone disease. Tc99m-methylene diphosphonate (MDP) and similar single-photon radiolabeled phosphate analogues are incorporated into the hydroxyapatite crystalline lattice and collagen matrix ${ }^{13}$. Uptake of MDP is a function of blood supply, rate of bone turnover or osteoblastic activity, quantity of mineralized bone, capillary permeability, fluid pressure and local acid/base balance. Bone scintigraphy is highly sensitive for osteogenic activity and allows for quick assessment of the entire skeleton. There are several limitations: bone scan findings depict derivative changes rather than the tumor itself; regression of disease is difficult to discern due to lingering radiotracer uptake in healing bone; and response assessment is confounded by the flare phenomenon, a major obstacle that can occur up to 12 weeks post effective treatment ${ }^{14}$.

In recent years, $\mathrm{F} 18$-sodium fluoride $(\mathrm{NaF})$ has re-emerged in the form of PET as a radiotracer for imaging bone metastases ${ }^{15}$. It was originally used for bone imaging with conventional gamma cameras applying a high energy collimator, yielding to poor resolution, and was then largely replaced by Tc99m-labeled bone tracers, which were more suitable for imaging with a conventional gamma camera ${ }^{16}$. The mechanism of $\mathrm{NaF}$ uptake is similar to that of the phosphonates. Advantages of NaF over MDP include its higher affinity for bone ${ }^{17}$, allowing for earlier imaging time with better image quality, which is also a product of the superior imaging characteristics of PET (figure 2). Several studies to date have suggested that NaF performs better than MDP for the assessment of osteoblastic metastases, particularly when combined with the anatomic information derived from CT. Notably, EvenSapir and colleagues compared planar bone scintigraphy, bone SPECT, NaF-PET, and NaF$\mathrm{PET} / \mathrm{CT}$ in patients with localized, high-risk or metastatic prostate cancer ${ }^{18}$. The reported 
sensitivity and specificity for detection of bone lesions was higher for NaF-PET/CT (100\% and $100 \%$, respectively) than for planar bone scanning (70\% and 57\%), SPECT (92\% and $82 \%$ ) or NaF-PET alone (100\% and $62 \%)$. These results appear to favor NaF-PET/CT over conventional bone scintigraphy; however, limitations of the study include the mixed patient population and lack of a true standard of reference.

Although NaF-PET is widely considered superior to MDP, no prospective studies have yet demonstrated an incremental benefit in staging or patient management; it is unclear if $\mathrm{NaF}$ will provide more meaningful information (possibly based on quantitative assessment) regarding treatment response and disease progression than the conventional bone scan. Additionally, it is uncertain whether quantitation of $\mathrm{NaF}$ uptake in bone lesions, using standardized uptake values (SUV), confers prognostic information. The information derived from a conventional planar bone scan can be reduced to a single quantitative parameter, termed the bone scan index (BSI) ${ }^{19}$, a measure of the fractional volume of skeletal tumor burden. BSI derived from conventional bone scans is prognostic of survival ${ }^{20,21,22}$, and its value as an indicator of response or progression is under investigation ${ }^{23}$. Manual BSI measurements are relatively reproducible, but are tedious to perform. An automated platform for BSI calculation that generates reproducible results within seconds was recently shown to correlate well with manually derived BSI scores ${ }^{24,25}$. With the increasing availability of NaF-PET it remains to be seen whether the BSI can be optimized even further by the three-dimensional and quantitative properties of PET imaging.

Further experience with NaF-PET is needed before it is able to supplant conventional single photon bone scans, which are less expensive and more widely available. With the recent decision of the Centers for Medicare \& Medicaid Services to reimburse sites participating in the National Oncologic PET Registry (NOPR) for NaF-PET scans, a wealth of data is expected to surface in the coming months and years.

\section{F18-FDG}

In 2008, the National Oncologic PET Registry (NOPR) published results on the clinical impact of FDG-PET ${ }^{26,27}$. The registry found that FDG-PET influenced management of prostate cancer patients in $35-45 \%$ of cases across clinical indications, similar to other cancers, despite the widely held notion that FDG is of limited value in prostate cancer. It is true that FDG-PET is of not the optimal modality for assessing organ-confined prostate cancer, owing to the low glycolytic activity of many tumors, and due to technical factors such as interference by physiologic, hyperplastic or inflammatory-type FDG uptake in the prostate, and high excreted activity in the adjacent urinary bladder. However, FDG-PET does play an important role in more advanced disease states ${ }^{28}$.

Schoder and colleagues compared the performance of FDG-PET/CT and conventional imaging in 91 patients with PSA relapse following prostatectomy ${ }^{29}$. The standard of reference included biopsy or clinical and imaging follow-up. PET was true positive in 28 of $91(31 \%)$ patients, showing isolated disease in the prostate bed $(n=3)$ or metastatic disease with $(n=2)$ or without $(n=23)$ simultaneous disease in the prostate bed. Mean PSA was higher in FDG-positive than in FDG-negative patients $(9.5 \pm 2.2$ versus $2.1 \pm 3.3 \mathrm{ng} / \mathrm{mL})$. 
PSA of $2.4 \mathrm{ng} / \mathrm{mL}$ and PSA velocity of $1.3 \mathrm{ng} / \mathrm{mL} / \mathrm{y}$ provided the best tradeoff between sensitivity $(80 \% ; 71 \%)$ and specificity $(73 \% ; 77 \%)$ of PET in a receiver operating curve analysis. Combination with other clinical parameters in a multivariate analysis did not improve disease prediction. In this study, there were only two patients in whom other imaging studies showed isolated local recurrence or metastatic disease.

Bone scanning, whether with NaF-PET or conventional Tc99m MDP agents, remains an indirect method of imaging bone metastases. Many sclerotic lesions detected on bone scan, including NaF-PET, are in reality dormant or treated. Furthermore, lytic or marrow-based lesions are not readily detectable on bone scan due to lack of bone turnover. FDG-PET, on the other hand, directly assesses tumor metabolism in bone. The value of FDG for assessment of bone metastases in castration resistant prostate cancer (CRPC) was specifically addressed by our group ${ }^{30}$. In this study, 43 patients underwent FDG-PET and bone scan prior to investigational therapies. Of 105 FDG-positive and MDP-negative lesions, 84 (80\%) eventually turned positive on followup bone scan. Survival correlated inversely with FDG-PET SUVmax (median survival 14.4 vs. 32.8 months if SUVmax > 6.10 vs. $\leq 6.10, \mathrm{p}=0.002)$, as well as with the BSI (14.7 vs. 28.2 months if BSI $>1.27$ vs. < $1.27 ; \mathrm{p}=0.004)$. Only SUVmax was an independent factor in multivariate analysis. A combination of SUVmax and a nomogram for progressive prostate cancer dichotomized patients into a high versus low risk group (median survival 14.4 vs. 34.6 months, $\mathrm{p}=.015$ ) that was more prognostic than either alone.

Clinical experience shows that FDG-PET can be applied for response assessment in patients with metastatic disease undergoing hormonal therapy or chemotherapy ${ }^{31,32}$. Preliminary data suggest that this is also possible with the choline tracers, however, larger prospective studies are lacking.

\section{Future Directions}

Molecular imaging probes that target antigens and receptors specifically expressed by prostate cancer cells may eventually be transformative biomarkers for disease management and drug development. Such PET agents are particularly relevant for navigating the biologic heterogeneity of advanced disease.

\section{Androgen Receptor (AR) Probes}

The AR signaling axis is implicated as a driving force in the development and progression of CRPC, justifying the need for novel antiandrogen therapies ${ }^{33}$. AR expression and binding capacity can be assessed non-invasively with F18-FDHT, an analog of dihydrotestosterone $(\mathrm{DHT})^{34}$. Since endogenous DHT (the primary AR ligand) competes with FDHT for AR binding, the tracer is most suitably applied in patients with castrate disease, which is characterized by low circulating testosterone levels $(<50 \mathrm{ng} / \mathrm{dL})^{35,36}$. In our experience with total-lesion analyses ${ }^{37}$ of paired FDG and FDHT-PET scans in metastatic CRPC, we have seen diverse patterns of uptake, including FDG/FDHT concordance, FDG predominance and FDHT predominance (figure 3). These unique phenotypes may have implications for risk stratification and personalization of therapeutic strategies. The potential role of FDHT-PET as a pharmacodynamic marker was recently demonstrated in the context of a therapeutic trial 
for a next-generation AR targeted therapy. In this phase 1-2 study of MDV3100, a competitive AR inhibitor, a clear-cut reduction in uptake ( 20-100\%) was seen in all 22 patients evaluated with FDHT-PET during therapy, with a suggestion of dose dependence and a saturation point prior to reaching the maximum tolerated dose ${ }^{38}$. Of note, these FDHT "responses" did not necessarily correlate with clinical response. At this time, it remains unclear if therapy-related modulation of FDHT uptake can predict clinical outcomes.

\section{Prostate Specific Membrane Antigen (PSMA) Probes}

FDHT uptake reflects AR ligand-receptor interaction, but the dynamics of downstream AR signaling are perhaps better captured by other molecular imaging probes. In particular, J591 is an antibody to an epitope on the extracellular domain of prostate specific membrane antigen (PSMA) that is promising for both imaging and radioimmunotherapy purposes ${ }^{39}$. PET imaging with J591 is proposed to reflect the downstream effects of AR inhibition, given that PSMA expression is downregulated by androgen administration and upregulated by androgen deprivation ${ }^{40,41}$. An in-human imaging trial of Zr89-labeled J591 (Zr89-DFOhuJ591 42 ) is currently underway at our institution, and a Cu64-labeled version of the antibody is in preclinical development ${ }^{43}$. Other PSMA targeted tracers are currently being developed by the group at John's Hopkins University ${ }^{44}$. Together, AR-axis imaging agents promise to increase our understanding of the biology and escape mechanisms of prostate cancer, hopefully with implications for novel therapies.

\section{Conclusion}

Multiple PET tracers are now available to aid in the detection and management of prostate cancer across the clinical spectrum of the disease. Prospective clinical imaging trials using various PET tracers, individually or in combination, rigorously controlled for clinical-state, therapy and well-defined clinical endpoints are needed to establish the optimal role of PET in prostate cancer, just as they are crucial in drug development ${ }^{45}$.

\section{Acknowledgements}

Support for this work came in part from ICMIC grant P50 CA086438-11 and Department of Defense grant PC071610

\section{REFERENCES}

1. Scher HI, Heller G. Clinical states in prostate cancer: Towards a dynamic model of disease progression. Urology. 2000; 55:323-327. [PubMed: 10699601]

2. Scher HI, Morris MJ, Kelly WK, et al. Prostate cancer clinical trial end points: "RECIST"ing a step backwards. Clin Cancer Res. 2005 Jul 15; 11(14):5223-5232. [PubMed: 16033840]

3. Bauman G, Belhocine T, Kovacs M, et al. 18F-fluorocholine for prostate cancer imaging: a systematic review of the literature. Prostate Cancer Prostatic Dis. 2012 Mar; 15(1):45-55. [PubMed: 21844889] This broad review of 18 F-fluorocholine underscores the need for rigorously controlled prospective imaging trials to better define the optimal role of PET in prostate cancer.

4. Souvatzoglou M, Weirich G, Schwarzenboeck S, et al. The sensitivity of [11C]choline PET/CT to localize prostate cancer depends on the tumor configuration. Clin Cancer Res. 2011 Jun 1; 17(11): 3751-3759. [PubMed: 21493868] This histopathologic correlation study emphasizes the limitations of PET in detecting small lesions and suggests that choline-PET is not a useful first line tool for diagnosing prostate cancer. 
5. Scher HI, Eisenberger M, D'Amico AV, et al. Eligibility and outcomes reporting guidelines for clinical trials for patients in the state of a rising prostate specific antigen: recommendations from the Prostate-Specific Antigen Working Group. J Clin Oncol. 2004; 22 3:537-556. [PubMed: 14752077]

6. Krause BJ, Souvatzoglou M, Tuncel M, et al. The detection rate of [11C]choline-PET/CT depends on the serum PSA-value in patients with biochemical recurrence of prostate cancer. Eur J Nucl Med Mol Imaging. 2008 Jan; 35(1):18-23. [PubMed: 17891394]

7. Giovacchini G, Picchio M, Scattoni V, et al. PSA doubling time for prediction of [(11)C]choline $\mathrm{PET} / \mathrm{CT}$ findings in prostate cancer patients with biochemical failure after radical prostatectomy. Eur J Nucl Med Mol Imaging. 2010 Jun; 37(6):1106-1116. [PubMed: 20306038]

8. Giovacchini G, Picchio M, Parra RG, et al. Prostate-Specific Antigen Velocity Versus ProstateSpecific Antigen Doubling Time for Prediction of 11C Choline PET/CT in Prostate Cancer Patients With Biochemical Failure After Radical Prostatectomy. Clin Nucl Med. 2012 Apr; 37(4):325-331. [PubMed: 22391699] This retrospective study showed a link between PSA kinetics and the detectability of recurrent disease on choline-PET. However, the included patients do not all meet the strict definition of "biochemical relapse", given evidence of metastatic disease on standard imaging.

9. Castellucci P, Fuccio C, Nanni C, et al. Influence of trigger PSA and PSA kinetics on 11C-Choline $\mathrm{PET} / \mathrm{CT}$ detection rate in patients with biochemical relapse after radical prostatectomy. $\mathrm{J}$ Nucl Med. 2009; 50:1394-1400. [PubMed: 19690023]

10. Schillaci O, Calabria F, Tavolozza M, et al. Influence of PSA, PSA velocity and PSA doubling time on contrast-enhanced (18)F-choline PET/CT detection rate in patients with rising PSA after radical prostatectomy. Eur J Nucl Med Mol Imaging. 2012 Apr; 39(4):589-596. Epub 2012 Jan 10. [PubMed: 22231016] This retrospective study adds to the growing evidence for a strong correlation between PSA kinetics and choline uptake in locally recurrent or metastatic disease. The included patients do not all qualify for the clinical state of "rising-PSA-only", owing to the presence of metastatic disease on standard imaging.

11. Bubendorf L, Schopfer A, Wagner U, et al. Metastatic patterns of prostate cancer: an autopsy study of 1589 patients. Hum Path. 2000; 31:578-583. [PubMed: 10836297]

12. Eisenhauer EA, Therasse P, Bogaerts J, et al. New response evaluation criteria in solid tumours: revised RECIST guideline (version 1.1). Eur J Cancer. 2009 Jan; 45(2):228-247. [PubMed: 19097774]

13. Subramanian G, McAfee JG. A new complex of 99mTc for skeletal imaging. Radiology. 1971; 99:192-196. [PubMed: 5548678]

14. Pollen JJ, Witztum KF, Ashburn WL. The flare phenomenon on radionuclide bone scan in metastatic prostate cancer. Am J Roentgenol. 1984; 142:773-776. [PubMed: 6230903]

15. Grant FD, Fahey FH, Packard AB, et al. Skeletal PET with 18F-fluoride: applying new technology to an old tracer. J Nucl Med. 2008 Jan; 49(1):68-78. [PubMed: 18077529]

16. Blau M, Nagler W, Bender MA. Fluorine-18: a new isotope for bone scanning. J Nucl Med. 1962 Jul.3:332-334. [PubMed: 13869926]

17. Segall G, Delbeke D, Stabin MG, et al. SNM practice guideline for sodium 18F-fluoride PET/CT bone scans 1.0. J Nucl Med. 2010 Nov; 51(11):1813-1820. [PubMed: 21051652]

18. Even-Sapir E, Metser U, Mishani E, et al. The detection of bone metastases in patients with high risk prostate cancer: 99mTc-MDP planar bone scintigraphy, single and multi field of view SPECT, 18F-fluoride PET and 18F-fluoride PET/CT. J Nuc Med. 2006; 47:287-297.

19. Imbriaco M, Larson SM, Yeung HW, et al. A new parameter for measuring metastatic bone involvement by prostate cancer: the bone scan index. Clin Cancer Res. 1998; 4:1765-1772. [PubMed: 9676853]

20. Sabbatini P, Larson SM, Kremer A, et al. Prognostic significance of extent of disease in bone in patients with androgen-independent prostate cancer. J Clin Oncol. 1999; 17:948-957. [PubMed: 10071289]

21. Meirelles GS, Schoder H, Ravizzini GC, et al. Prognostic value of baseline [18F] fluorodeoxyglucose positron emission tomography and 99mTc-MDP bone scan in progressing metastatic prostate cancer. Clin Cancer Res. 2010 Oct 25.

22. Ulmert D, Kaboteh R, Fox JJ, et al. A Novel Automated Platform for Quantifying the Extent of Skeletal Tumour Involvement in Prostate Cancer Patients Using the Bone Scan Index. Eur Urol. 
2012 Jan 27. This study offers evidence for validation of an automated program designed to evaluate planar bone scans and quantify the bone scan index.

23. Dennis ER, Jia X, Mezheritskiy IS, et al. Bone scan index: a quantitative treatment response biomarker for castration-resistant metastatic prostate cancer. J Clin Oncol. 2012 Feb 10; 30(5): 519-524. [PubMed: 22231045] Qualified biomarkers are important for streamlining the approval of novel therapies in prostate cancer. This study provides early evidence for the bone scan index as a response indicator in CRPC and supports further exploration of bone scintigraphy as an imaging biomarker.

24. Sadik M, Suurkula M, Höglund P, et al. Improved classifications of planar whole-body bone scans using a computer-assisted diagnosis system: a multicenter, multiple-reader, multiple-case study. J Nucl Med. 2009 Mar; 50(3):368-375. [PubMed: 19223423]

25. Ulmert D, Kaboteh R, Fox JJ, et al. A Novel Automated Platform for Quantifying the Extent of Skeletal Tumour Involvement in Prostate Cancer Patients Using the Bone Scan Index. Eur Urol. 2012 Jan 27.

26. Hillner BE, Siegel BA, Shields AF, et al. Relationship between cancer type and impact of PET and PET/CT on intended management: findings of the national oncologic PET registry. J Nucl Med. 2008 Dec; 49(12):1928-1935. Epub 2008 Nov 7. [PubMed: 18997054]

27. Hillner BE, Siegel BA, Liu D, et al. Impact of positron emission tomography/computed tomography and positron emission tomography (PET) alone on expected management of patients with cancer: initial results from the National Oncologic PET Registry. J Clin Oncol. 2008 May 1; 26(13):2155-2161. Epub 2008 Mar 24. Erratum in: J Clin Oncol. 2008 Sep 1;26(25): 4229. [PubMed: 18362365]

28. Jadvar H. FDG PET in Prostate Cancer. PET Clin. 2009 Apr 1; 4(2):155-161. [PubMed: 20161051]

29. Schöder H, Herrmann K, Gönen M, et al. 2-[18F]fluoro-2-deoxyglucose positron emission tomography for the detection of disease in patients with prostate-specific antigen relapse after radical prostatectomy. Clin Cancer Res. 2005 Jul 1; 11(13):4761-4769. [PubMed: 16000572]

30. Meirelles GS, Schoder H, Ravizzini GC, et al. Prognostic value of baseline [18F] fluorodeoxyglucose positron emission tomography and 99mtc-mdp bone scan in progressing metastatic prostate cancer. Clin Cancer Res. 2010 Oct 25.

31. Morris MJ, Akhurst T, Osman I, et al. Fluorinated deoxyglucose positron emission tomography imaging in progressive metastatic prostate cancer. Urology. 2002; 59:913-918. [PubMed: 12031380]

32. Morris MJ, Akhurst T, Larson SM, et al. Fluorodeoxyglucose positron emission tomography as an outcome measure for castrate metastatic prostate cancer treated with antimicrotubule chemotherapy. Clin Cancer Res. 2005; 11:3210-3216. [PubMed: 15867215]

33. Clegg NJ, Wongvipat J, Joseph JD, et al. ARN-509: A Novel Antiandrogen for Prostate Cancer Treatment. Cancer Res. 2012 Mar 15; 72(6):1494-1503. [PubMed: 22266222] This preclinical study offers proof of principle for ARN-509 as a therapeutic agent in castration-sensitive and castration-resistant forms of prostate cancer. The authors indicate a higher therapeutic index than clinically available antiandrogens including MDV3100, in part demonstrated by competitive binding assays versus F18-FDHT.

34. Liu A, Dence CS, Welch MJ, Katzenellenbogen JA. Fluorine-18-labeled androgens: radiochemical synthesis and tissue distribution studies on six fluorine-substituted androgens, potential imaging agents for prostatic cancer. J Nucl Med. 1992; 33:724-734. [PubMed: 1569482]

35. Larson SM, Morris M, Gunther I, et al. Tumor localization of 16beta-18F-fluoro-5alphadihydrotestosterone versus $18 \mathrm{~F}-\mathrm{FDG}$ in patients with progressive, metastatic prostate cancer. $\mathbf{J}$ Nucl Med. 2004 Mar; 45(3):366-373. [PubMed: 15001675]

36. Dehdashti F, Picus J, Michalski JM, et al. Positron tomographic assessment of androgen receptors in prostatic carcinoma. Eur J Nucl Med Mol Imaging. 2005 Mar; 32(3):344-350. [PubMed: 15726353]

37. Fox JJ, Autran-Blanc E, Morris MJ, et al. Practical approach for comparative analysis of multilesion molecular imaging using a semiautomated program for PET/CT. J Nucl Med. 2011 Nov; 52(11):1727-1732. [PubMed: 21984797] This methodological paper introduces a systematic 
approach for performing total lesion analyses on multi-tracer and longitudinal PET comparisons, based on the paradigm of paired FDG and FDHT-PET imaging in metastatic CRPC patients.

38. Scher HI, Beer TM, Higano CS, et al. Antitumour activity of MDV3100 in castration-resistant prostate cancer: a phase 1-2 study. Lancet. 2010; 375:1437-1446. [PubMed: 20398925]

39. Pandit-Taskar N, O'Donoghue JA, Morris MJ, et al. Antibody mass escalation study in patients with castration-resistant prostate cancer using 111In-J591: lesion detectability and dosimetric projections for 90Y radioimmunotherapy. J Nucl Med. 2008 Jul; 49(7):1066-1074. [PubMed: 18552139]

40. Wright GL Jr, Grob BM, Haley C, et al. Upregulation of prostate-specific membrane antigen after androgen-deprivation therapy. Urology. 1996 Aug; 48(2):326-334. [PubMed: 8753752]

41. Kuroda K, Liu H, Kim S, et al. Docetaxel down-regulates the expression of androgen receptor and prostate-specific antigen but not prostate-specific membrane antigen in prostate cancer cell lines: implications for PSA surrogacy. Prostate. 2009 Oct 1; 69(14):1579-1585. [PubMed: 19575420]

42. Holland JP, Divilov V, Bander NH, et al. 89Zr-DFO-J591 for immunoPET of prostate-specific membrane antigen expression in vivo. J Nucl Med. 2010 Aug; 51(8):1293-1300. [PubMed: 20660376]

43. Evans MJ, Smith-Jones PM, Wongvipat J, et al. Noninvasive measurement of androgen receptor signaling with a positron-emitting radiopharmaceutical that targets prostate-specific membrane antigen. Proc Natl Acad Sci U S A. 2011 Jun 7; 108(23):9578-9582. Epub 2011 May 23. [PubMed: 21606347] Preclinical study supporting the utility of Cu64-J591 PET for quantifying androgen receptor signaling in prostate cancer.

44. Mease RC, Dusich CL, Foss CA, et al. N-[N-[(S)-1,3-Dicarboxypropyl]carbamoyl]-4[18F]fluorobenzyl-L-cysteine, [18F]DCFBC: a new imaging probe for prostate cancer. Clin Cancer Res. 2008 May 15; 14(10):3036-3043. [PubMed: 18483369]

45. Scher HI, Morris MJ, Basch E, Heller G. End points and outcomes in castration-resistant prostate cancer: from clinical trials to clinical practice. J Clin Oncol. 2011 Sep 20; 29(27):3695-3704. Epub 2011 Aug 22. [PubMed: 21859988] Important review on the challenges faced in prostate cancer management and drug development, with emphasis on the use of biomarkers as clinical endpoints. 


\section{Key Points}

- C11-choline and F18-choline PET are useful for restaging of prostate cancer; uptake appears to be correlated with PSA kinetics in patients with recurrent disease.

- FDG-PET and NaF-PET are valuable in advanced disease, especially for assessing bone metastases.

- $\quad$ FDHT-PET non-invasively assesses the status of the androgen receptor (AR) expression in the setting of castrate disease.

- Prostate specific membrane antigen (PSMA) targeted PET tracers are proposed to reflect the effects of AR inhibition on downstream signaling, and are in the early stages of clinical development.

- Prospective, rigorously controlled, clinical imaging trials are needed to establish the optimal role of PET in prostate cancer. 


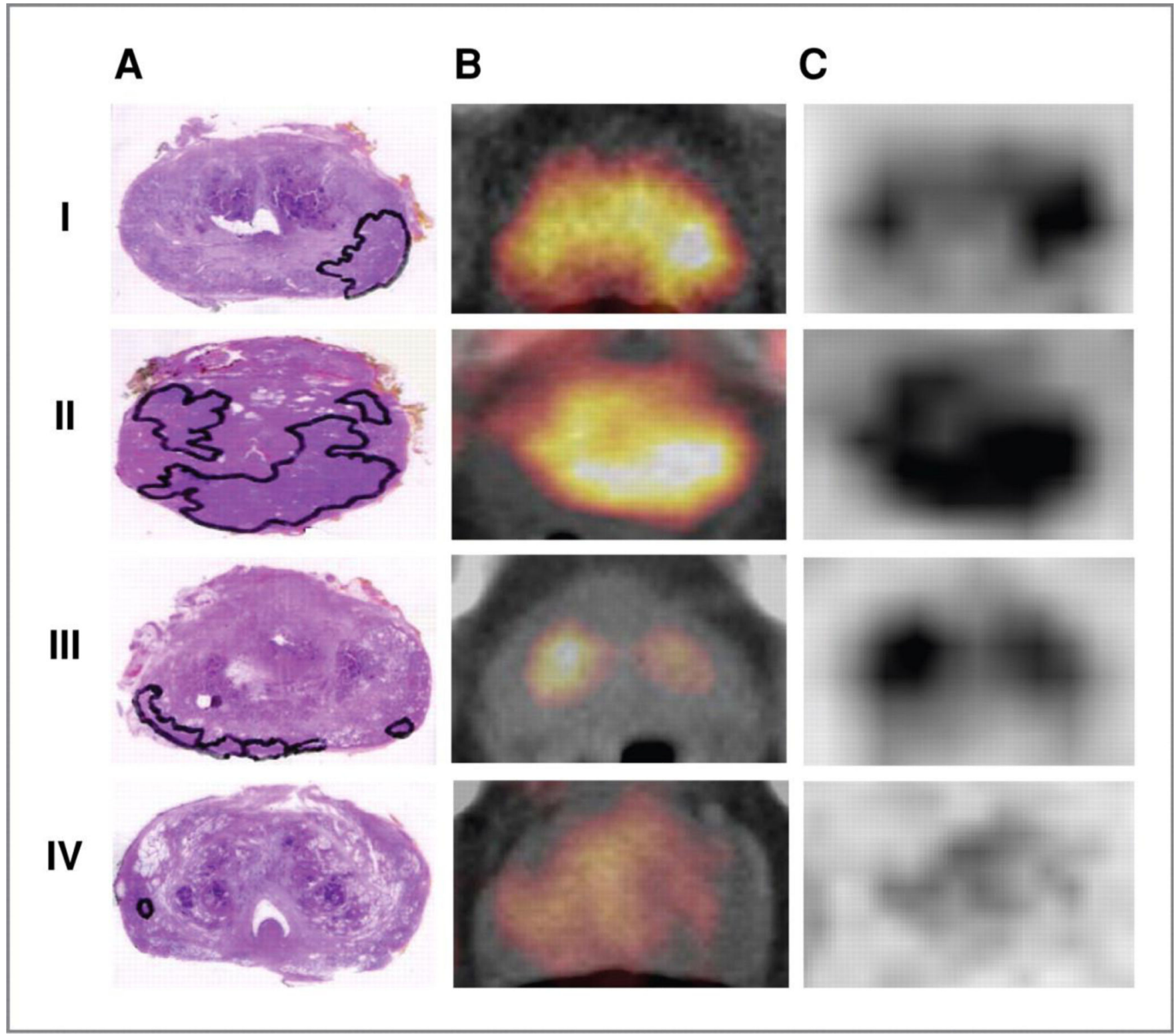

Figure 1. The sensitivity of C11-Choline PET/CT for localizing prostate cancer (PCa) depends on tumor configuration

Histology specimen (A), PET/CT fused image (B), and PET image (C) of the respective slices in the 4 (I-IV) different tumor configuration forms observed. The tumor is outlined in the histologic specimen. In the first and in the second row unifocal (form I) and multifocal (form II) PCa are shown exhibiting intense C11-choline uptake (SUVmax = 5.6 in I; SUVmax = 7.1 in II). In the third and the fourth row PCa with a rind-like shaped growth pattern (form III) and a small sized focus of cancer (form IV) are shown that are not visualized in the corresponding PET images (SUVmax $=5.7$ located in BPH in III and SUVmax $=3.5$ located in normal prostate tissue in IV). Reproduced with permission from Souvatzoglou M et al. Clin Cancer Res 2011;17:3751-3759 
A

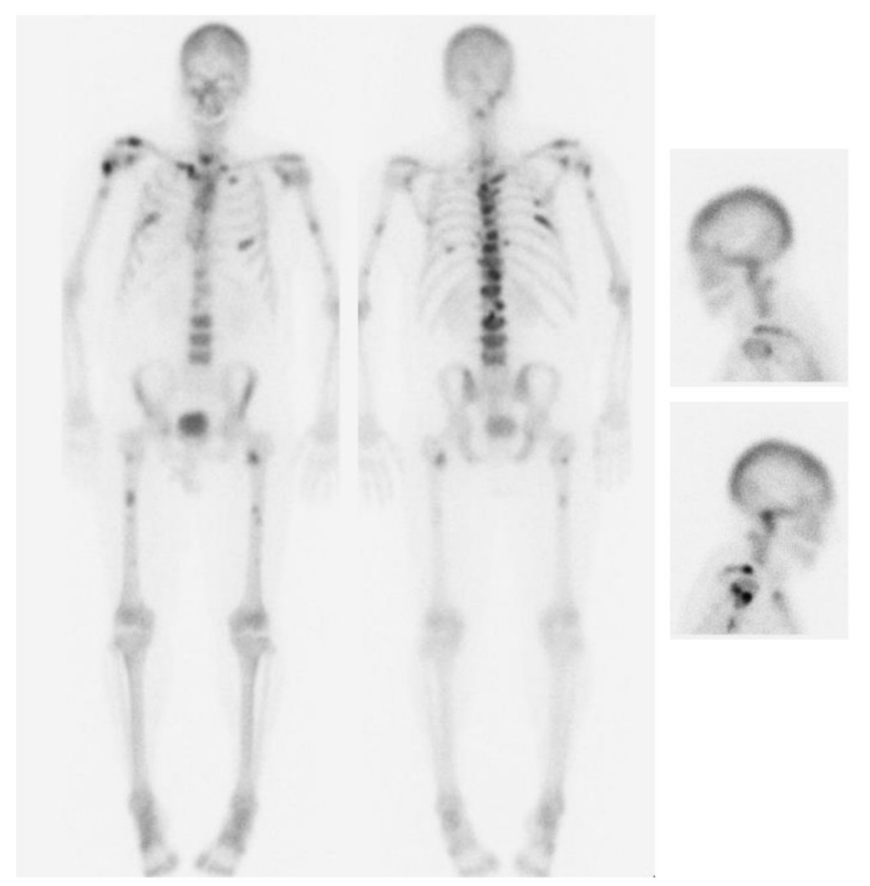

B

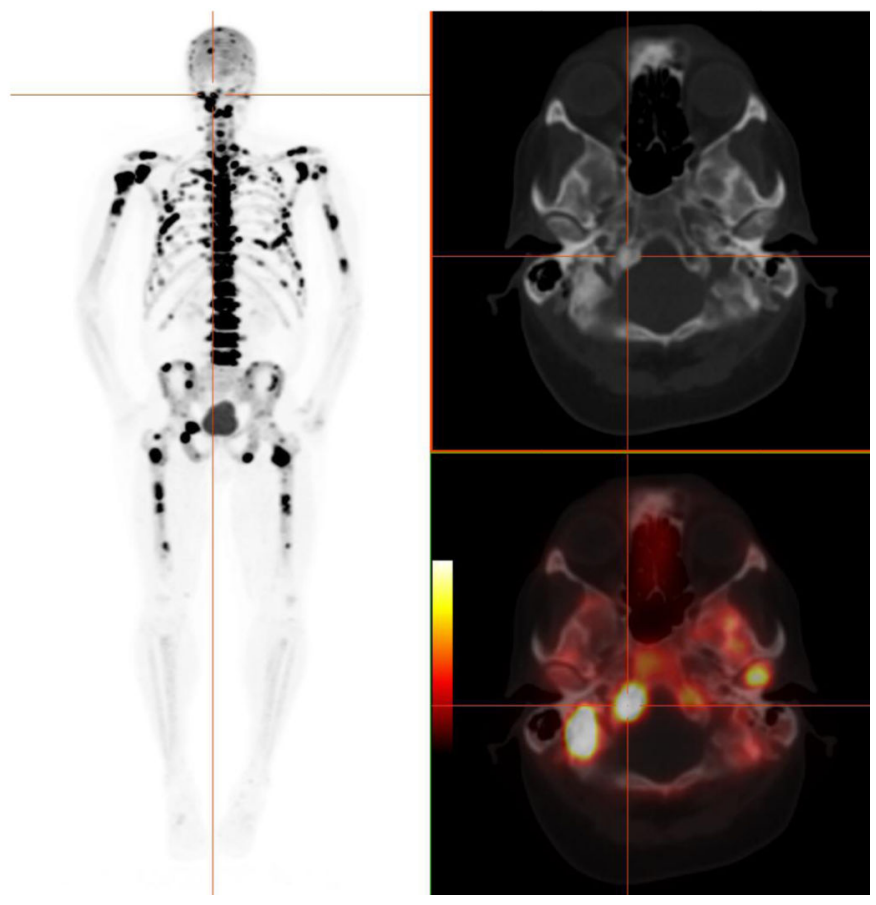

Figure 2. Enhanced depiction of bone metastases with NaF-PET in a 64-year-old male with CRPC

A. Tc99m-MDP whole body images and lateral spot views of the head demonstrate multiple osseous metastases in the axial and appendicular skeleton. B. NaF-PET/CT MIP, axial CT and fused axial PET/CT images in the same patient show superior detection of numerous metastatic lesions with exquisite demonstration of skull base metastases that are poorly visualized on MDP scan. 
A

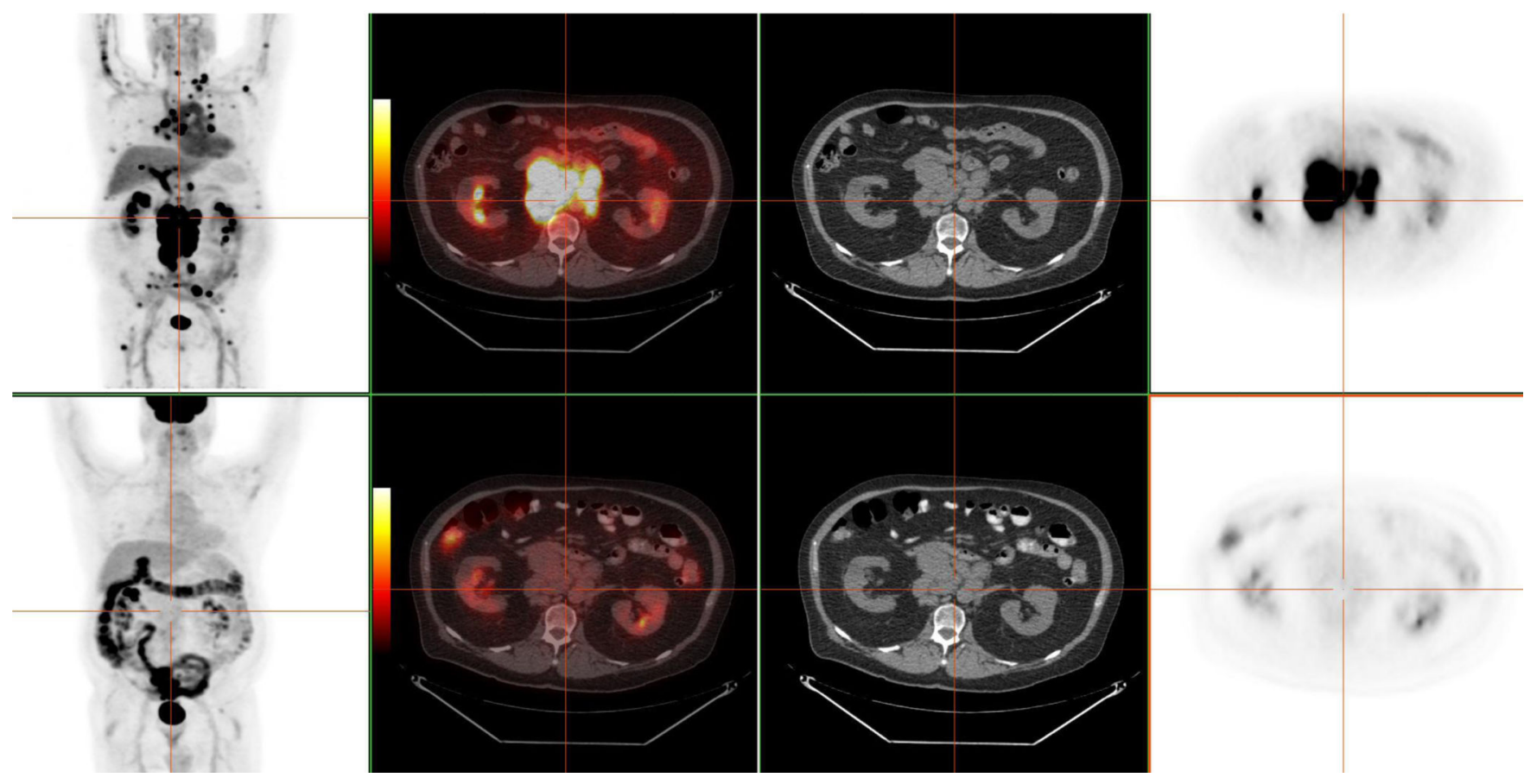

$\mathrm{B}$
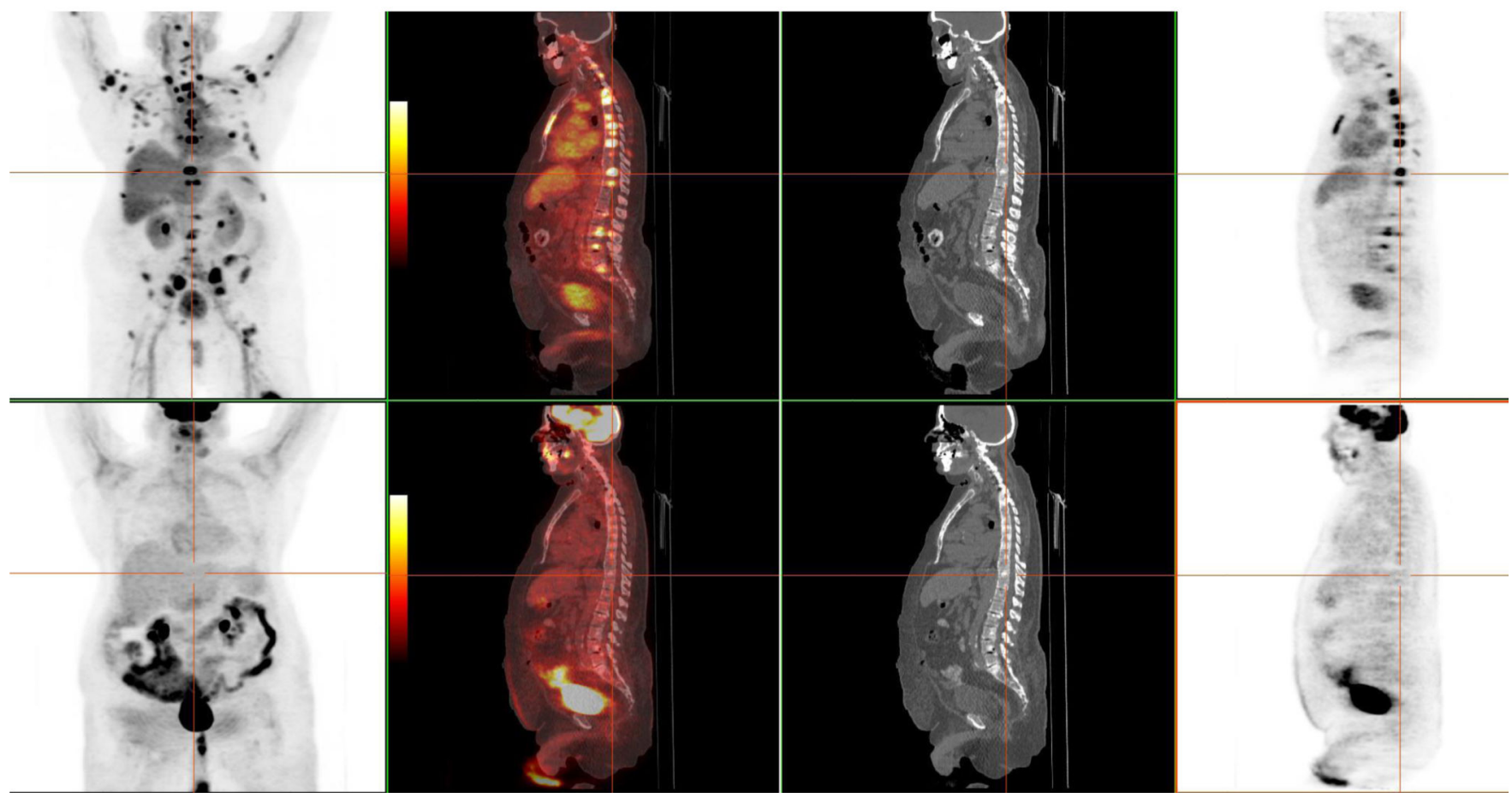

Figure 3.

A: FDHT-predominant nodal disease in a 67-year-old male with CRPC and PSA of 789 $\mathbf{n g} / \mathbf{m l}$. FDHT (top) and FDG (bottom) MIP, fused axial, CT axial and PET axial images 
demonstrate marked discordance in avidity of extensive retroperitoneal nodal metastases. MIP images also demonstrate multiple discordantly FDHT-positive bone lesions.

B: FDHT-predominant bone disease in a 69-year-old male with CRPC and PSA of 213 $\mathbf{n g} / \mathbf{m l}$. FDHT (top) and FDG (bottom) MIP, fused sagittal, CT sagittal and PET sagittal images demonstrate marked discordance in avidity of bone metastases. 


\section{Table 1}

PET Tracers for Clinical Imaging of Prostate Cancer

\begin{tabular}{|c|c|c|}
\hline Tracer & Isotope Half-Life & Mechanism \\
\hline F18-FDG & $110 \mathrm{~min}$ & $\begin{array}{l}\text { Analog of glucose; reflects the increased glycolytic activity of tumors (Warburg effect); FDG is } \\
\text { trapped in cells via GLUT transport and irreversible HK phosphorylation }\end{array}$ \\
\hline C11 -Choline & $20 \mathrm{~min}$ & $\begin{array}{l}\text { Substrate for phospholipid synthesis in cell membranes, transmembrane signaling, lipid and } \\
\text { cholesterol transport and metabolism; choline kinase is upregulated in tumors }\end{array}$ \\
\hline F18-Choline & $110 \mathrm{~min}$ & Same as for C11-choline \\
\hline C11-Methionine & $20 \mathrm{~min}$ & $\begin{array}{l}\text { Naturally occurring amino acid; reflects increased amino acid transport, to a lesser degree also } \\
\text { protein synthesis related to tumor cell proliferation and turnover }\end{array}$ \\
\hline C11-Acetate & $20 \mathrm{~min}$ & $\begin{array}{l}\text { Naturally occurring metabolite; converted to acetyl-CoA and incorporated into cholesterol and } \\
\text { fatty acids ; fatty acid synthetase and acetyl-CoA carboxylase are oncogenic enzymes } \\
\text { upregulated in prostate cancer }\end{array}$ \\
\hline F18-FACBC & $110 \mathrm{~min}$ & $\begin{array}{l}\text { Synthetic l-leucine analog; reflects increased amino acid transport as prerequisite for protein } \\
\text { synthesis }\end{array}$ \\
\hline Sodium F18-Fluoride & $110 \mathrm{~min}$ & $\begin{array}{l}\text { Reflects increased osteoblastic activity by slow exchange of fluoride ions with hydroxyapatite } \\
\text { crystals, forming fluoroapatite }\end{array}$ \\
\hline F18-FDHT & $110 \mathrm{~min}$ & $\begin{array}{l}\text { Androgen receptor expression and binding capacity; AR is upregulated in castrate resistant } \\
\text { disease }\end{array}$ \\
\hline Zr89-DFO-huJ591 & $78.4 \mathrm{hr}$ & Monoclonal antibody to epitope on external domain of PSMA \\
\hline
\end{tabular}

FDG $=2$-[18F]fluoro-2-deoxyglucose; GLUT = Glucose Transporter; HK = Hexokinase; FACBC = Anti-1-Amino-3-[18F]fluorocyclobutane-1carboxylic acid; FDHT = 16 $\beta$ - $[18 \mathrm{~F}]-$ Fluoro-5a-dihydrotestosterone; PSMA = Prostate Specific Membrane Antigen 